\title{
Cosmetic Lateral Canthoplasty: Lateral Canthoplasty to Lengthen the Lateral Canthal Angle and Correct the Outer Tail of the Eye
}

\author{
Soo Wook Chae ${ }^{1}$, Byung Min Yun ${ }^{2}$ \\ ${ }^{1}$ BY Plastic Surgery Clinic, Seoul; ${ }^{2}$ Department of Plastic and Reconstructive Surgery, Jeju National University, Jeju, Korea
}

There are many women who want larger and brighter eyes that will give a favorable impression. Surgical methods that make the eye larger and brighter include double eyelidplasty, epicanthoplasty, as well as lateral canthoplasty. Double eyelidplasty produces changes in the vertical dimension of the eyes, whereas epicanthoplasty and lateral canthoplasty create changes in the horizontal dimension of the eyes. Epicanthoplasty, a surgical procedure which enlarges the eye horizontally, is performed at the inner corner of the eye, whereas lateral canthoplasty enlarges the outer edge of the eye. In particular, if the slant of the palpebral fissure is raised and the horizontal dimension of the palpebral fissure is short, adjusting the slant of the palpebral fissure through lateral canthoplasty can achieve an enlargement of eye width and smoother features. Depending on the patient's condition, even better results can be achieved if this procedure is performed in conjunction with other procedures, such as double eyelidplasty, epicanthoplasty, eye roll formation surgery, fat graft, and facial bone contouring surgery. In this paper, the authors will introduce in detail their surgical method for a cosmetic lateral canthoplasty that lengthens the lateral canthal angle and corrects the outer tail of the eyes, in order to ease the unfavorable impression.

Keywords Cosmetics / Lateral canthoplasty
Correspondence: Soo Wook Chae BY Plastic Surgery Clinic, Wookyung Bldg. 5th Fl., 466 Apgujeong-ro, Gangnam-gu, Seoul 06015, Korea Tel: +82-2-541-5522

Fax: +82-2-545-8743

E-mail: bydoctor@naver.com

Received: 9 Jun $2016 \bullet$ Revised: 1 Jul $2016 \bullet$ Accepted: 6 Jul 2016

pISSN: 2234-6163 • elSSN: 2234-6171 • http://dx.doi.org/10.5999/aps.2016.43.4.321 • Arch Plast Surg 2016;43:321-327

No potential conflict of interest relevant to this article was reported.

\section{INTRODUCTION}

Many women desire to have larger and brighter eyes. Many patients wish to obtain a larger eye shape by lengthening the lateral canthal angle and, at the same time, a brighter eye by correcting the raised outer tail of the eyes via lateral canthoplasty. If the horizontal dimension of the palpebral fissure is short and the lateral canthus is located higher than the medial canthus, it can give a stubborn, angry and unfavorable impression.

There have been many misconceptions about lateral canthoplasty, including that (1) after lateral canthoplasty, the patient's eye eventually returns to its preoperative state as time passes, (2) a single operation is insufficient, and so multiple operations are necessary, (3) a natural drooping of the outer tail of the eye occurs with aging so that there is no need for surgery, and that (4) in order to prevent postsurgical complications such as shrinkage of the lengthened lateral canthal angle, patients must keep their eyes open, without closing them, and keep their outer eye area stretched out. These misconceptions are so prevalent that not only patients but also plastic surgeons tend to avoid cosmetic lateral canthoplasty.

Through experience with thousands of cases, the authors first 
presented "Cosmetic lateral canthoplasty which lengthens the lateral canthal angle and simultaneously corrects the raised the outer tail of the eyes using periosteal fixation" at the 10th Meeting of the Association for Research on Minimal Invasive Plastic Surgery: MIPS in April 2008. After that, the authors have given numerous presentations on surgical methods at various conferences, symposiums and training lectures. To make the eyes appear larger and brighter and to achieve a favorable impression, the authors conducted lateral canthoplasty, which lengthens the lateral canthal angle and lowers the raised outer tail of the eyes. In this paper, by introducing the authors' own method, the authors intend to provide a guide and surgical tips to perform an effective cosmetic lateral canthoplasty.

\section{ANATOMY OF THE LATERAL CANTHAL AREA}

There are three components of the lateral canthal area under the skin; the lateral palpebral raphe (LPR), superficial lateral palpebral ligament (SLPL), and deep lateral palpebral ligament (DLPL). The lateral ends of the superior and inferior orbicularis oculi muscles interlace at the lateral commissure and form the LPR.

The lateral palpebral ligament is divided into the SLPL and the DLPL. The SLPL extends from the lateral ends of tarsal plate to the periosteum of lateral orbital rim. The DLPL extends from the lateral ends of tarsal plate deep into the origin of the SLPL to Whitnall's tubercle on the zygomatic bone inside the orbital margin. It is located deeper than the SLPL. The DLPL is attached to the lateral orbital tubercle (Whitnall's tubercle). Whitnall's tubercle was located $2.9-0.8 \mathrm{~mm}$ inside of the orbital rim of the zygoma $[1]$.

When performing lateral canthoplasty, the SLPL must be incised and dissected to release the lateral canthus, enabling an ef- fective surgery. As the DLPL is deeply located and difficult to find, there is no need to expend effort detaching or incising it.

\section{SURGICAL PROCEDURES}

\section{Design}

The design of the surgery is very simple. Following the crease around the lateral canthus (A), the end point of the incision line (B) is marked (Figs. 1,2). At this time, the end point should not go past the lateral wall of the bony orbit. Marking the lowest point of the bony orbit will help to find a fixation point during surgery.

\section{Local infiltration}

First, anesthetic eye drops (Alcaine $0.5 \%$, proparacaine hydrochloride) are applied to the eye. Using a 30 gauge $1 / 2$ inch nee-

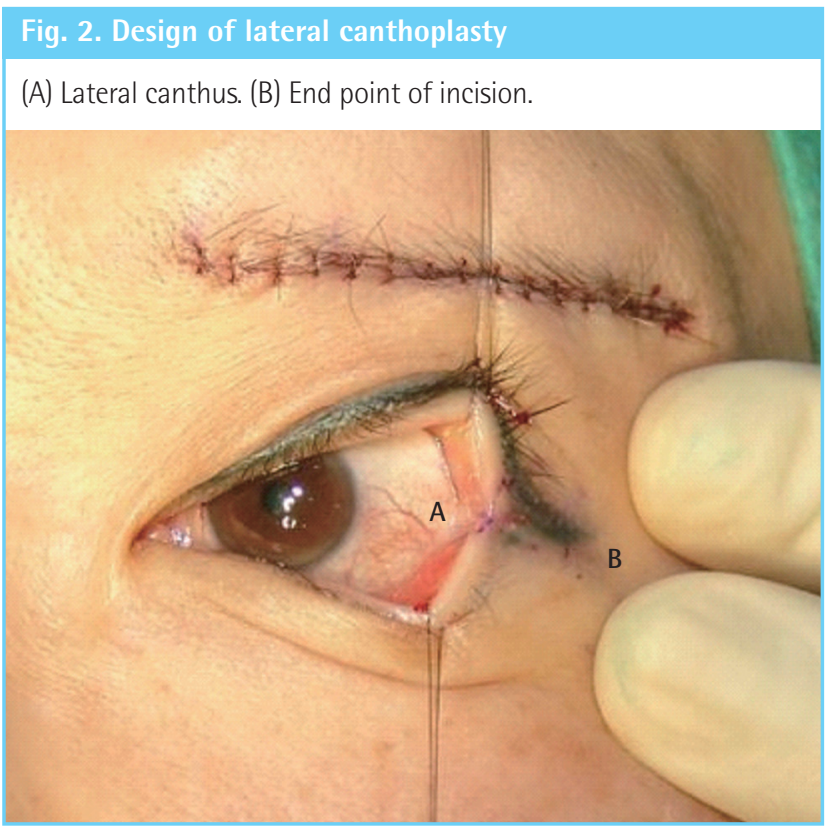

\section{Fig. 1. Schematic drawing of the operation, left eye}

A \& $A^{\prime}$, lateral canthus; $B$, end of incision; $A^{\prime}-B$, new lateral canthus; $\downarrow$, lowering of the slant of the palpebral fissure; the shaded area, enlargement after lateral canthoplasty.
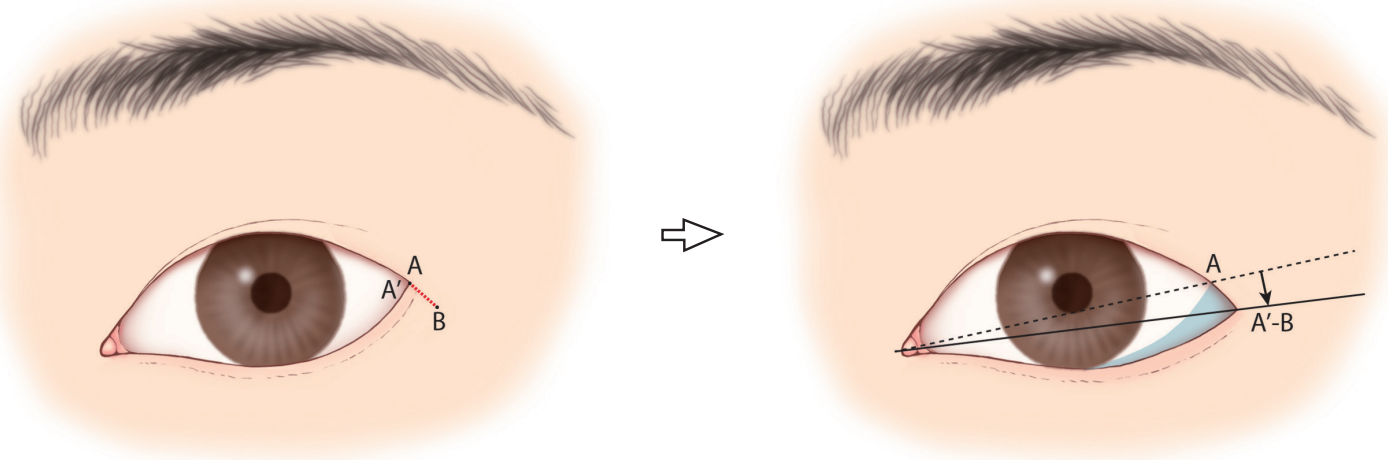
dle, an anesthetic containing 1:100,000 epinephrine mixed with a 1:1 solution of $0.5 \%$ bupivacaine hydrochloride and saline solution is injected.

\section{Incision and dissection}

Before beginning the operation, traction sutures are applied to the upper and lower eyelids. A skin incision is then carried out from point $\mathrm{A}$ to point $\mathrm{B}$ (Fig. 3A). After incision and dissection of the LPR and the SLPL, the lower lid is pulled lightly in order to check whether it has become sufficiently released. At this point, care should be taken not to damage the DLPL through excessively deep dissection (Fig. 3B). A critical zone delineates a circle with a radius of $0.5 \mathrm{~cm}$, and its center is located $30^{\circ}$ inferiorly and laterally, and $2.5 \mathrm{~cm}$ from the lateral canthus [2].

Using a 6-0 nylon suture, canthopexy is performed at the periosteum and lower lid (Fig. 4). In order to produce a firm fixation, suturing is done at two places, and the point of fixation should be made within $3 \mathrm{~mm}$ of the lower lid margin. When affixing to



\section{Fig. 4. Canthopexy}

Canthopexy to (A) the periosteum and (B) lower eyelid.

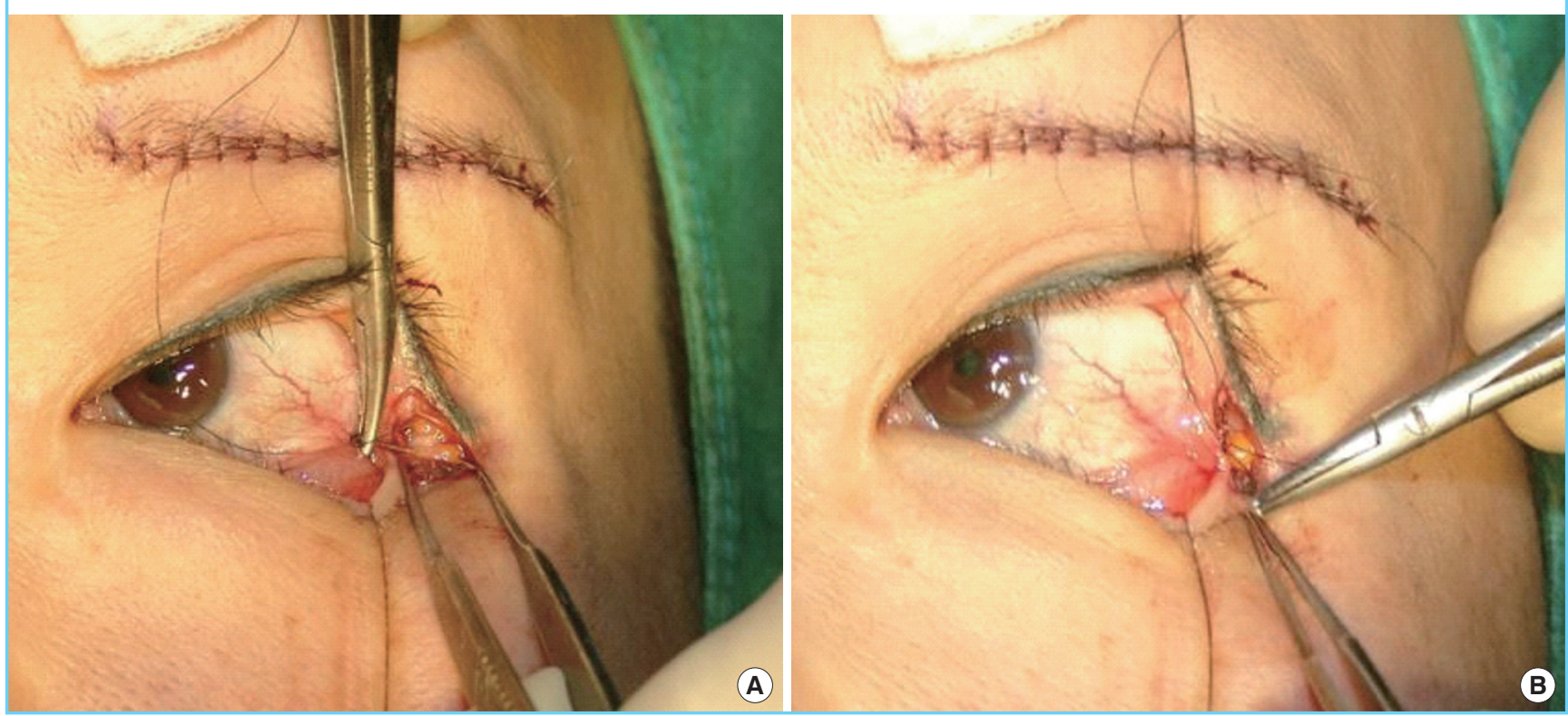




\begin{abstract}
Fig. 5. Case 1
Preoperative and postoperative photographs of a 51-year-old female patient. A sub-brow lift, non-incisional double eyelidplasty, and lateral canthoplasty were performed. (A-D) Preoperative photographs. (A-C) Preoperative frontal view, three-quarter right side view, three-quarter left side view (D) view of patient looking upwards. (E-H) Photos six months postoperatively. (E-G) Postoperative frontal view, three-quarter right side view, three-quarter left side view $(\mathrm{H})$ view of patient looking upwards. When the postoperative eyelash tattoo was compared with the preoperative eyelash tattoo, the outcomes of the surgery were evident.
\end{abstract}


the periosteum, a firm fixation should be placed at the inner side of the bony orbit's lateral wall in order to prevent complications such as eversion of the lower eyelid after surgery.

\section{Skin closure}

A skin suture was placed using 7-0 black silk. As the area of surgery is close to the eyeball, soft thread should be used in order to reduce irritation to the eyeball.

\section{Factors to consider after surgery}

After surgery, oral antibiotics and ophthalmic ointment, as well as three kinds of eye drops (antibiotics, steroids, and artificial tears) are prescribed. Steroid eye drops should only be used for a short period of time when the palpebral conjunctiva is swol- len. Care must be taken not to open or rub the wound.

While the patient may wash their face or take a light shower after removing the stitches, they should wait for three weeks before going to a sauna or swimming pool and before wearing contact lenses.

\section{Postoperative progress}

The stitches are removed five to seven days after surgery. Within one month after surgery, swelling is reduced by more than $90 \%$. Palpebral conjunctival swelling and bloodshot eyes mostly disappear within three weeks. While the incision line can appear red for about two to three months, as time passes, it blends in with the surrounding skin color and is no longer noticeable. 


\section{Fig. 6. Case 2}

Preoperative and postoperative photographs of a 23-year-old female patient. Non-incisional double eyelidplasty and lateral canthoplasty were performed together. $(A, B)$ Preoperative frontal view, three-quarter right side view photos. (C, D) Frontal view, three-quarter right side view photos one month postoperatively.


\section{Fig. 7. Case 3}

Photograph of a 35-year-old female patient, postoperatively. Only lateral canthoplasty was performed. $(A, B)$ frontal side preoperatively, left side three-quarter view photos. (C, D) 5 years and 4 months postoperatively, frontal view, three-quarter left side view photos.
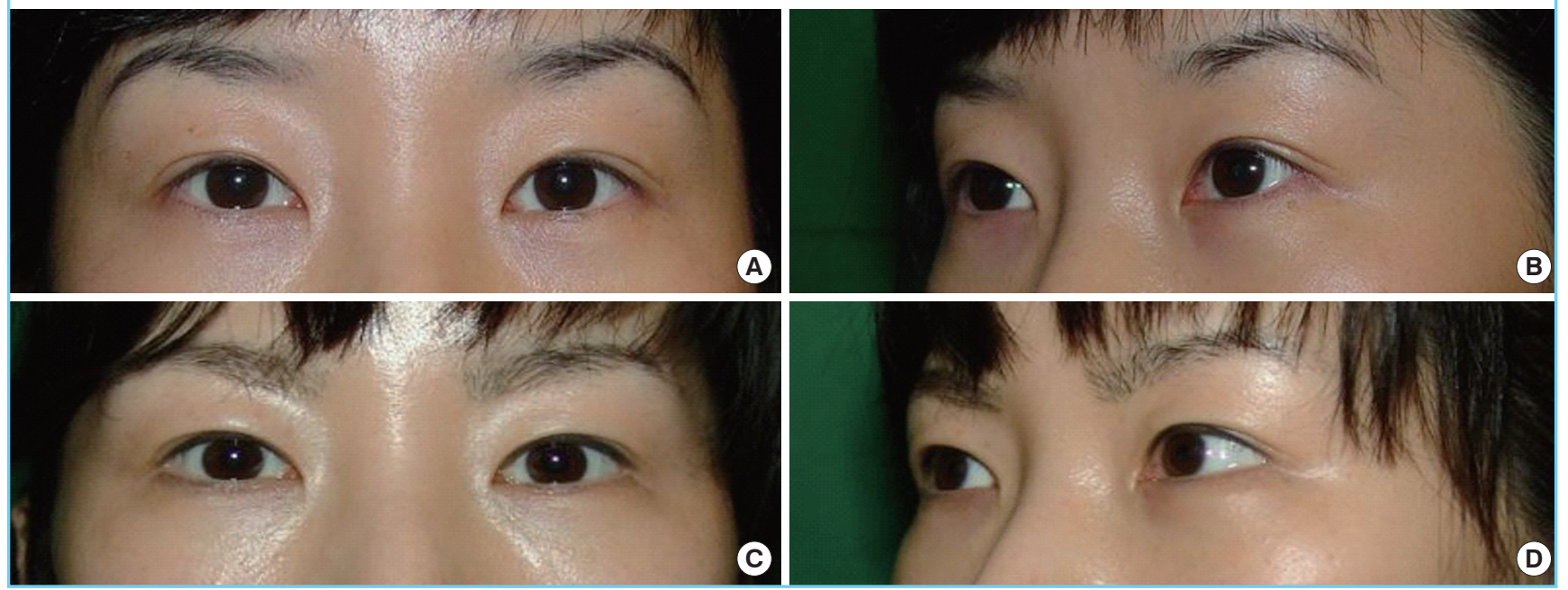

\section{Clinical cases}

\section{Case 1}

A sub-brow lift, non-incisional double eyelidplasty, and lateral canthoplasty were performed on a 51-year-old female patient (Fig. 5).

\section{Case 2}

Non-incisional double eyelidplasty and lateral canthoplasty were performed on a 23-year-old female patient (Fig. 6).

\section{Case 3}

A 35-year-old female patient who underwent lateral canthoplas- ty (Fig. 7).

\section{DISCUSSION}

The horizontal dimension of the palpebral fissure in Koreans is $26.8 \pm 1.9 \mathrm{~mm}$ in men and $26.1 \pm 1.9 \mathrm{~mm}$ in women, and the average slant of the palpebral fissure for men and women over ten years old was $7.9 \pm 2.4$ degrees in men and $8.8 \pm 2.3$ degrees in women, with a greater degree of incline showing in women than men [3]. Because many persons have a short horizontal dimension and raised outer tail of the eyes, there are many patients who want cosmetic lateral canthoplasty. 
Until recently, lateral canthoplasty had been performed mainly on patients with lower lid malposition such as retraction, as well as lid laxity, ectropion, and entropion. However, plastic surgeons as well as patients had been reluctant to perform or undergo cosmetic lateral canthoplasty due to the many instances of extensive scarring or lack of effectiveness in cosmetic applications for the lengthening of the lateral canthal angle.

Shin et al stated the principles and guidelines for performing cosmetic lateral canthoplasty as follows: (1) the continuity of the lower eyelid margin should be preserved, (2) the contact surface should be kept fittingly between the bulbar conjunctiva and palpebral conjunctiva, (3) the eyelashes should be saved, and (4) the patient's orbital condition should be more than $4 \mathrm{~mm}$ from the orbital rim to the lateral canthal angle in exophthalmometry [4].

In the authors' experience, the lateral canthoplasty is successful if (1) there is plenty of space between the lateral canthus and the lateral bony orbital wall, giving enough leeway for performing lateral canthoplasty, (2) the eye slants upward, (3) the eye is exophthalmic rather than enophthalmic, (4) and the size of the eye is large. On the other hand, surgery tends to be less effective when (1) there is a small amount of space between the lateral canthus and the lateral bony orbital wall, making canthoplasty difficult, (2) the eye is sunken in or (3) the eye corner slants downwards, (4) there is a severe blepharochalasia, covering the lateral canthus, (5) the volume of the bony orbit is small, or (6) the eye is small and (7) lacks a double eyelid.

An effective lateral canthoplasty can be achieved by performing horizontal lengthening and vertical lengthening of the lateral canthal angle at the same time.

If the lateral canthus is simply incised and sutured, the effect of the lateral canthus lengthening will be insignificant. In instances when the inner palpebral conjunctiva is exposed, a red palpebral conjunctiva is visible, and when the skin flap is raised, scarring may occur on the outer area of the lateral canthus. If incision to the outer area is performed, avoiding the lateral canthus, complications may occur such as the disappearance of the lower lid eyelashes during dissection, visible scarring of the lower eyelid margin, or scarring in the shape of a notch appearing at the starting point of the incision of the lateral end of the upper eyelid.

Depending on the postoperative presence of a gray line defect, we can classify lateral canthoplasty by cases in which there is no gray line defect, where the gray line defect is limited to the upper eyelid, where it is limited to the lower eyelid, and where it is present on both the upper and lower eyelid.

It would be ideal to obtain good results from lateral canthoplasty without having a gray line defect, but in actuality, this kind of lateral canthoplasty leads to poor surgical results.
If a gray line defect remains at the lower eyelid, lower eyelid continuity is destroyed and the eye shape appears unnatural or the palpebral conjunctiva is exposed, making it appear red. In addition, the lateral rectus capsulopalpebral fascia is attached at the lower eyelid tarsus, functioning as a lid retractor. Since a defect in this can affect the functioning of the eyelid, it should be avoided as much as possible. For example, if a gray line defect appears at both upper and lower lids, as in the von Ammon method, there is a high possibility that scar contracture will occur, so that the shape of the lateral canthus becomes rounded or the palpebral conjunctiva is pulled taut, becoming exposed and appearing red.

While it is ideal not to have any gray line defect, if its occurrence cannot be avoided, it is best for the defect to appear at the lateral end of the upper eyelid. This is because, while there are ciliaeyelashes on the upper eyelid, there normally are not any eyelashes within a few millimeters of the lateral end, and any eyelash defects caused by lateral canthoplasty can be sufficiently hidden by the surrounding eyelashes and slightly drooping skin. It is important that the continuity of the lower eyelid be preserved. There should be no defect of the eyelashes on the lower eyelid.

The authors' own methods comprise canthotomy, cantholysis, and canthopexy. Not only are these methods effective for lengthening the lateral canthal angle, it is possible to correct the slant of the palpebral fissure at the same time. While a gray line defect appears at the upper eyelid lateral end, it can be sufficiently hidden and does not become a major problem.

During lateral canthoplasty, because lengthening of the lateral canthal angle is ineffective in conducting cantholysis or canthotomy and suturing through a simple skin incision, canthopexy is performed simultaneously. Canthopexy not only has good results in lengthening the lateral canthal angle, it can also correct the slant of the palpebral fissure.

Canthopexy is performed using a 6-0 nylon suture, and at this time, fixation should be done within $3 \mathrm{~mm}$ from the margin of the lower eyelid in order for the fixation to be effective. Generally, the tarsal plates are semilunar shaped. They are of 3 types: symmetric, medially skewed, and laterally skewed. The skewed types are more common than the symmetric type. The width of the upper lid tarsal plate is $9.3 \mathrm{~mm}$ at its center, whereas the width of the lower lid tarsal plate is about $4.6 \mathrm{~mm}$ at its center. The width of the medial $1 / 4$ and the lateral $1 / 4$ comes to no more than $3.9 \mathrm{~mm}$ and $3.8 \mathrm{~mm}$, respectively [5]. As the lateral rectus capsulopalpebral fascia and the lateral canthal tendon are connected at the lateral aspect of the tarsus [6], canthopexy should be conducted within $3 \mathrm{~mm}$ from the lower eyelid margin in order to have solid results.

Taking the vectors of the canthopexy into consideration, fixa- 
tion should be performed inside the bony orbit in order to prevent postoperative eversion of the lower eyelid's outer side.

There are three important techniques for creating a natural eye shape after lateral canthoplasty: first, beginning the incision precisely at the lateral canthus; second, performing a fixation at the inside of the bony orbit when carrying out canthopexy; and third, precisely matching the lateral canthal angle when suturing the skin.

As the eyeball is round, it is important that the lower eyelid and the eyeball remain in contact with each other after canthoplasty. When canthopexy is performed, fixation should be performed at the inner area of the bony orbit, and if the lateral canthal angle is matched well when the skin is sutured, then after surgery the eyeball and the lower eyelid will be in proper contact and the eye shape will become natural after surgery. There are congenital cases, though they are rare, in which the eyeball and lower eyelid do not come into contact with each other, and since lower eyelid surgery performed to correct entropion can result in the failure of the eyeball and lower eyelid to contact each other, the surgeon must take this into consideration through consultation and medical interviews.

Postoperatively, complications can occur such as conjunctival hemorrhage, conjunctiva edema, and, uncommonly, dellen. Even in severe cases, however, recovery is possible through two to three weeks of conservative treatment.

The lateral canthal angle borders the eyeball and is the place where tears flow. If it is not properly managed, there is a high chance that wound dehiscence could occur. For three weeks after surgery, care should be taken to avoid any impact to the site, and not to open or rub the wound at all. The occurrence of dehiscence may result in fish mouth-shaped scarring, which can be easily corrected through a simple scar revision once scar maturation has completed.

\section{CONCLUSIONS}

While lateral canthoplasty by itself can provide sufficient results as a type of eye surgery, it has a complementary character in that the results are even more prominent in eyes with double eyelids. Changes mainly appear in the outer eye area. This surgery is appropriate for patients who want a larger and brighter eye shape and want to achieve a favorable impression by lowering the raised the outer tail of their eyes.

If this operation is conducted through the selection of suitable patients and the surgeon is familiar with the surgical methods, the operation can be safe, reliable and provide good results. Depending on the status of the patient, simultaneously performing another operation that can make the eye large and bright, such as double eyelidplasty or epicanthoplasty, can provide even better results.

\section{REFERENCES}

1. Hwang K, Nam YS, Kim DJ, et al. Anatomic study of the lateral palpebral raphe and lateral palpebral ligament. Ann Plast Surg 2009;62:232-6.

2. Hwang K, Lee DK, Lee EJ, et al. Innervation of lower eyelid in relation to blepharoplasty and midface lift: clinical observation and cadaveric study. Ann Plast Surg 2001;47:1-7.

3. Park DH, Back BS, Nahai F. Cosmetic and reconstructive oculoplastic surgery. Seoul: Gunja Publishing Co.; 2009.

4. Shin YH, Hwang K. Cosmetic lateral canthoplasty. Aesth Plast Surg 2004;28:317-20.

5. Kim YS, Hwang K. Shape and heights of tarsal plates. J Craniofac Surg 2016;27:496-7.

6. Kakizaki H, Zako M, Nakano T, et al. Microscopic findings of lateral tarsal fixation in Asians. Ophthal Plast Reconstr Surg 2008;24:131-5. 